\title{
- L'eau, l'aménagement du territoire et le développement durable
}

\author{
- Water, town and country planning and sustainable development -
}

\author{
par J. Dausset \\ Prix Nobel de Médecine, \\ Président de l'Académie de l'eau.
}

\section{L'eau est partout présente et doit donc faire partie intégrante de l'aménagement du territoire.}

L'eau est présente partout. Nécessaire à la vie des hommes, elle sert aussi à évacuer les rejets de leur habitat tout comme elle est indispensable à leurs activités agricoles, industrielles ou commerciales et à leurs loisirs. Son rôle est aussi important pour la flore, la faune et les rivières tant pour leur quantité que pour leur qualité ou encore pour la production de l'énergie et la navigation. Son impact sur la santé des hommes, leur environnement, et sur la qualité de la vie, est également très fort à cause de sa présence et de ses interactions dans tous les domaines. Elle est en effet universelle et vivante. Son cycle la met en contact avec la terre entière (ruissellement et érosion, infiltration, pollution ...) et la regroupe dans les fleuves, les nappes et la mer. Il la purifie enfin grâce à l'évaporation mue par le soleil et son passage dans l'atmosphère. Elle est donc indissociable de l'aménagement du territoire, du développement économique et des activités humaines aussi bien dans les agglomérations que dans les zones rurales.

Ces liens sont connus mais, jusqu'à encore très récemment, les conférences internationales sur l'eau incitaient seulement les gestionnaires du cycle de l'eau à se concerter et pas à élargir leur cercle interne à celui de l'aména- gement. Cette globalisation est aujourd'hui préconisée après la conférence de Rio de 1992 et celle d'Istanbul de 1996 sur l'habitat.

L'Académie de l'eau qui regroupe les spécialistes de l'eau avec ceux des sciences de la vie et de la terre et de l'aménagement a pour mission principale de mettre en évidence les rapports entre l'eau et tous les autres domaines pour mieux protéger l'environnement et la qualité de vie. Elle est donc tout à fait consciente de la nécessité d'expliciter les contraintes entre l'eau et l'aménagement du territoire. C'est pourquoi elle a engagé depuis près de trois ans une réflexion sur l'eau, la ville et l'urbanisme et plus récemment, une autre sur l'eau, le monde rural et l'aménagement du territoire avec l'appui financier des Agences de l'eau.

\section{L'EAU EN ZONE URBAINE}

Cette réflexion sur l'eau, la ville et l'urbanisme s'est traduite par un Symposium que l'Académie a organisé avec I'UNESCO en avril 1997. Il a réuni plus de 300 participants venus de 50 pays, des responsables de l'eau, des urbanistes, des médecins, des sociologues, ... et des élus pour discuter de "l'eau, de la ville et de l'urbanisme" à partir de monographies de 23 villes du monde. La conférence a adopté la "Déclaration de Paris" qui recommande un rapprochement entre eau et aménagement urbain s'appuyant sur une politique de développement durable et

Water is everywhere and therefore strongly linked to town and country planning, development and all human activities. The Academie de l'Eau has decided to establish the links between water and the other sectors in order to protect the environment and life quality. These reflections lead to some recommandations : a global, sparing, environment respectful, long-lasting management, showing solidarity. Two examples are given : the water management in the urban area along the Marne river and the case of rural zones. 
propose la création d'un système d'aide à la gestion urbaine. Son objectif serait d'abord de l'utiliser comme un outil de communication entre tous les partenaires et les citoyens sur des cas concrets, puis de progresser grâce à des échanges et des transferts entre villes à partir d'un réseau thématique relié aux réseaux de villes existants.

Ces propositions complèteraient celles d'une autre conférence tenue aussi à I'UNESCO sur "l'eau au 2le siècle" en mars 1996 à l'initiative du "Mouvement universel pour la responsabilité scientifique" (MURS) et de l'Académie.

Celle-ci a poursuivi la mise en œuvre de ces recommandations avec des villes françaises comme Paris, Lyon, Lille, Marseille.

Nancy, Nantes... et quelques villes étrangères qui estiment que l'organisation d'une politique de développement durable autour des cinq principes suivants est un excellent moyen pour établir une large concertation entre tous les secteurs de l'agglomération :

- une gestion globale associant l'ensemble des ressources face aux besoins des hommes et de leurs activités, - une gestion économe évitant les gaspillages.

- une gestion préservant l'environnement et la qualité de vie,

- une gestion solidaire pour l'homme faite à son profit et avec lui.

- une gestion durable conservant dans le temps les moyens mis en æuvre grâce à leur maintenance, à leur renouvellement et à leur maintien en bon état.

Les idées majeures sont de considérer l'aménagement comme un tout en prenant en compte tous ses aspects et de le mettre en ouvre de façon à assurer la meilleure qualité de vie aux habitants aujourd'hui et demain, en les y associant étroitement, et aussi d'organiser cette politique nouvelle autour du cycle de l'eau qui, étant présent partout, constitue une trame idéale pour la concertation nécessaire entre tous les acteurs. En France, les "Schémas d'aménagement et de gestion de l'eau". les SAGE peuvent être un excellent cadre pour cette nouvelle politique.

La méthodologie proposée a l'intérêt d'obliger les responsables de l'aménagement urbain. les gestionnaires de l'eau de la zone d'études et les usagers à travailler ensemble avec les associations représentant les citoyens pour établir trois documents :

- une photographie de la situation urbaine.

- une analyse des défauts et dysfonctionnements constatés et des remèdes à appliquer.

- les indicateurs de suivi de la politique décidée constituant ensemble un tableau de bord de la gestion urbaine.
La Région Ile de France qui a décidé une telle réflexion fin juin 1997 avec l'appui de l'Académie a choisi de l'appliquer au SAGE Marne aval afin de préparer un outil de gestion transposable à d'autres secteurs de l'agglomération puis à sa totalité.

La zone d'étude est située le long de la Marne, de Meaux à Charenton et Saint-Maur. Sur les quelque $1000 \mathrm{~km}^{2}$ de cette zone vivent 965000 habitants sur 245 $\mathrm{km}^{2}$ urbanisés. Elle englobe la ville nouvelle de "Marne la Vallée" et constitue un secteur où l'urbanisation se poursuit fortement avec une prédominance marquée pour l'habitat individuel (près des $3 / 4$ des habitants). Elle concerne une centaine de communes. Sur la surface totale de la zone, plus de $50 \%$ sont encore occupés par des bois, des cultures et des espaces verts, un quart par l'habitat ( $23 \%$ en individuel. $2 \%$ en collectif). Les voiries et voies ferrées représentent $13 \%$ et les équipements urbains le solde. C'est donc une zone rurale pour l'amélioration du cycle de l'eau mais aussi pour l'environnement et la qualité de la vie avec notamment :

- l'aménagement de la coulée verte de la Marne, en liaison étroite avec la réhabilitation de la rivière,

- les zones de loisirs de Vaires et de Torcy ainsi que le Parc des Boucles de la Marne,

- le parc d'attractions Disneyland,

- des voiries douces, comme celles de Joinville-le-Pont, etc.

C'est pourquoi cette zone a paru toute désignée pour la mise en place d'un outil expérimental de gestion du développement durable qui pourrait profiter de la réunion dans la Commission locale de l'eau des mêmes partenaires que ceux associés à l'étude dans un "Comité de suivi" (Etat, administration, gestionnaires, usagers, associations).

L'Académie de l'eau a prévu, en accord avec la région, que les premiers résultats de cette réflexion soient présentés en 1998 à la douzaine des autres villes françaises et étrangères qui travaillent aussi sur le développement durable pour permettre des échanges d'expérience.

Parallèlement ces résultats feront l'objet d'échanges avec les villes du monde grâce au réseau thématique préconisé dans la déclaration de Paris rassemblant au départ une quarantaine de villes

\section{II $\square$ LE CAS DES ZONES RURALES}

L'Académie de l'eau a entrepris avec l'Académie d'agriculture une réflexion similaire sur ces zones. Ce sont les parties du territoire excluant les agglomérations de plus 
de 50000 habitants et les centres moins peuplés mais jouant un rôle majeur sur le plan régional. Elles excluent aussi les zones montagneuses vides d'habitants. En France, elles recouvrent 85 à $90 \%$ de la superficie totale avec $300000 \mathrm{~km}^{2}$ en culture et $140000 \mathrm{~km}^{2}$ en forêt.

On peut diviser en deux groupes distincts :

- les zones prospères grâce essentiellement à l'activité agricole (cultures de toutes sortes, vigne, horticulture, élevage, légumes...) et à ses dérivés industriels ainsi que celles où le moteur du développement est le tourisme en complément de l'agriculture,

- les zones en difficulté.

Dans ces deux secteurs, l'eau sous toutes ses formes joue un rôle majeur notamment pour l'irrigation et l'assainissement des terres et/ou pour apporter aux populations locales et aux touristes la qualité de vie la meilleure possible. Elle contribue aussi dans les zones de montagne à la production d'électricité.

Le cycle de l'eau doit donc être pris en compte dans la politique d'aménagement du territoire à prévoir.

1. - Dans les zones où l'agriculture est prospère et constitue le facteur principal de développement comme à titre d'exemple dans la Beauce, la Brie, la Bretagne, on constate la transformation de l'agriculture traditionnelle en une agriculture intensive où est recherché le rendement maximum grâce aux engrais, aux pesticides et à une mécanisation très poussée ou grâce à la mise en place d'élevages industriels. Ainsi le Bassin Parisien produit $50 \%$ du blé tendre français et $44 \%$ des céréales, et la Bretagne $52 \%$ de la production de porcs. Il en résulte beaucoup de problèmes pour l'environnement notamment : - l'augmentation très forte des nitrates dans les nappes qui dépassent la limite de $50 \mu \mathrm{g} /$ litre dans plus de $25 \%$ de celles-ci,

- l'augmentation des pesticides (Simazine, Atrazine...) dans l'eau vers des teneurs dangereuses pour la santé,

- la production forte de méthane et de gaz à effet de serre et la forte consommation d'énergie,

- la réduction des zones humides et de la biodiversité,

- la réduction des populations rurales avec le groupement des terres et la mécanisation poussée.
Ces dysfonctionnements qui perturbent l'environnement et la santé, comme l'affaire de la vache folle, peuvent être réduits ou supprimés grâce à une concertation organisée entre gestionnaires de l'eau, agriculteurs et aménageurs du territoire ainsi qu'avec une réorientation des aides. Les opérations Fertimieux ou Irrimieux montrent que des résultats significatifs peuvent être obtenus par la concertation. La promotion de produits biologiques garantis par un label mais plus chers, comme pour certains fromages, est une voie intéressante. L'expérience montre qu'elle est acceptée par les consommateurs.

Dans les zones où c'est le tourisme qui est moteur, les liens entre eau et environnement doivent être développés également pour préserver la qualité des sites comme celles des plages et des eaux marines ou celle des lacs menacés par l'eutrophisation. Enfin, la préservation de la qualité de vie des touristes rejoint les efforts à mener grâce à la concertation dans les agglomérations.

2. - Dans les zones en difficulté, la réduction du nombre des habitants menace les infrastructures ainsi que l'entretien des rivières d'où des risques de dégradation du rôle de ces zones comme collecteur et producteur d'eau de qualité pour l'aval. L'aménagement du territoire doit donc chercher à y fixer une partie de la population et éviter leur afflux vers les villes.

Comme dans les zones urbaines, ces réflexions ont montré l'importance de la concertation entre spécialistes de l'eau, aménageurs et défenseurs de l'environnement avec les usagers, qu'ils soient agriculteurs, responsables du tourisme... et les citoyens.

Là aussi une politique du développement durable analogue à celle esquissée pour les villes est un moyen puissant pour que cette concertation se réalise.

L'Académie de l'eau a développé dans une communication à la Conférence de Paris du 19 au 20 mars, les actions en cours et celles prévues pour que s'élabore grâce au développement durable un aménagement du territoire exemplaire où l'eau joue un rôle moteur. Elle insiste également sur l'importance d'une bonne analyse des réactions des citoyens à partir d'enquêtes sociologiques analogues à celles qu'elle a réalisées sur une dizaine de villes. 